\title{
Sex chromosome evolution in Simulium erythrocephalum (Diptera: Simuliidae)
}

\author{
R. J. Post*
}

\author{
School of Biological Sciences, University of East \\ Anglia, Norwich, England
}

\begin{abstract}
The sex chromosomes of Simulium erythrocephalum show a pattern of serial differentiation by the linkage of inversions to either $\mathrm{X}$ or $\mathrm{Y}$. Two inversion systems, $I L-A$ and $I S-B+I+G$ are maintained in stable polymorphism with sex differences in frequency throughout southern England. These inversions are linked to the sex chromosomes such that males are more likely to be heterozygous. Heterozygosity and homozygosity for inversions $I L-A$ and $I S-B$ respectively seem to be associated with speed of larval development and are probably selected.
\end{abstract}

\section{INTRODUCTION}

Sex chromosome heteromorphy is known from many groups of animals and plants, and in most cases it is the Y-chromosome which is the smaller element. Presumably such systems evolved from morphologically identical and freely recombining sex chromosomes. Muller (1914) envisaged this process as a consequence of the perpetual heterozygosity of the $\mathrm{Y}$-chromosome, implying that it alone was able to degenerate by the accumulation of recessive deleterious mutations, such as small deletions and other gene dysfunctions, because these will be masked by the equivalent functional locus on the X-chromosome. Specific numerical models of this process assume that recombination is already suppressed between the $\mathrm{X}$ and $\mathrm{Y}(\mathrm{Nei}, 1970$; Charlesworth, 1978). Thus attention focuses on mechanisms which restrict recombination between the sex chromosomes, and structural rearrangement by inversion is one such mechanism.

Size difference between the sex chromosomes of Simuliidae is virtually unknown, and in most species they are indistinguishable in the polytene nucleus. Furthermore, males are usually chiasmate (Rothfels and Mason, 1975) and it is therefore probable that sex is determined primarily by a single locus, or at most a very short length of chromosome. However, many studies have revealed species in which the sex chromosomes

\footnotetext{
* Present address: Department of Medical Entomology, Liverpool School of Tropical Medicine, Pembroke Place, Liverpool L3 5QA, U.K
}

have become differentiated by the linkage of inversions. Such inversions may be fixed or polymorphic on either of the sex chromosomes, or occur on both but at different frequencies (Post 1982a). The purpose of this article is to describe the behaviour of several such inversions in the blackfly Simulium erythrocephalum, and to attempt to identify the selective forces which act upon them and hence might lead to the restriction of recombination between the $\mathrm{X}$ and $\mathrm{Y}$.

\section{MATERIALS AND METHODS}

Simulium (Boophthora) erythrocephalum (De Geer, 1776) is a small blood-sucking fly with immature stages restricted to rivers where the larvae are filter feeders. In central Europe $S$. erythrocephalum can be a serius agricultural pest and man-biter. Although it is also common in certain lowland British rivers, here it never seems to present much of a nuisance (Post, 1982b).

Post (1983) presented size frequency histograms for a series of regular samples of larvae taken from the River Tiffey at Carlton Forehoe in Norfolk during 1978. It is possible not only to identify each of the five annual generations from these histograms, but also to separate samples of larvae attaining their last instar earlier or later than the majority within that generation. A number of other rivers in southern England were occasionally sampled, and collection data are presented in table 1 .

Larvae were fixed in the field in freshly mixed Carnoy's solution and stored at $0^{\circ} \mathrm{C}$. Only large 


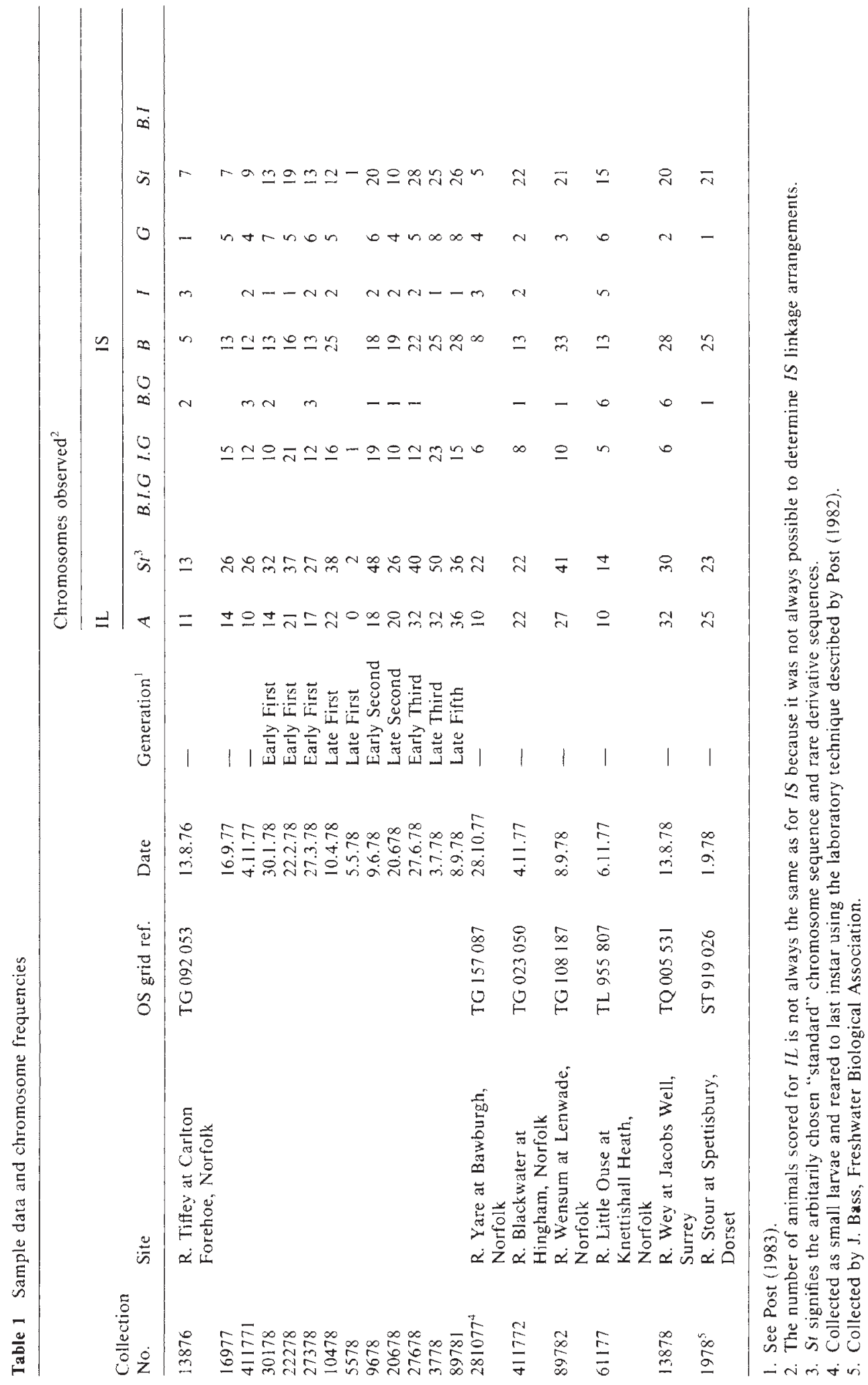


larvae (last or penultimate instar) were used for chromosome preparation. Larvae were split open along the ventral surface of the abdomen and the silk glands removed (see Puri, 1925). The silk glands were softened, on a microscope slide, with a few drops of 80 per cent acetic acid for five minutes and then stained in lacto-propionic orcien for four minutes. The silk glands were washed in 60 per cent acetic acid and the epithelium peeled off the jelly-like gland contents. The epithelium was restained for four minutes in lacto-propionic orcein and squashed in 60 per cent acetic acid. The preparation was made permanent, after examination, by the freeze-dry technique using euparol (Darlington and La Cour, 1976).

Whenever possible the larval body was subsequently stained with Feulgen (Darlington and La Cour 1976) to reveal the gonadial rudiment. The shape of the gonadial rudiment is a very reliable indicator of sex (Puri 1925), but in some larvae the rudiment could not be observed, including the whole of samples 55781 and 89782 . This was usually the result of technical problems in dissection and staining, but also the rudiments can be underdeveloped in parasitized larvae. Feulgen stained testes were squashed and mounted in 60 per cent acetic acid for observation of male meiosis.

\section{GENERAL KARYOTYPE}

Testicular meiosis was found to be chiasmate (fig. 1) demonstrating that recombination occurs in males of $S$. erythrocephalum, as it does in most species of blackfly (Rothfels and Mason 1975).

The haploid complement of $S$. erythrocephalum is $n=3$, which is also the number of elements observed in the polytene nucleus, because synapsis of the homologues is almost complete (fig. 2).

From a total of 510 specimens sampled from seven sites (table 1) 34 obvious chromosomal rearrangements were observed across all six chromosome arms, but the only rearrangements to show any sex linkage are some of those on the first (longest) chromosome. It is concluded therefore that it is this chromosome which forms the sex chromosome pair. The observed sex-linked rearrangements can be separated into two classes: those which are limited to one sex chromosome or the other (including $I L-Q . R . S . T$ and $I S-P+H$ ), and those which occur on both $\mathrm{X}$ and $\mathrm{Y}$ but at different frequencies (including $I L-A$ and $I S-B+$ $I+G)$.

\section{SEX CHROMOSOME DIFFERENTIATION}

On examination of the polytene chromosomes from the larval silk glands all males were found to possess a short non-pairing region at the base of chromosome arm $I S$ (and here labelled $I S$-split: fig. 3). Furthermore one of the bands within this non-pairing region was consistently heterozygous for intensity of staining. In females this band was homozygous for the fainter staining condition, and the bivalent was fully synapsed throughout its length, with a single exception. One female was found to possess $I S$-split, but it could not be determined if this was also associated with heterozygosity for the band staining. Band heteromorphy is not uncommon in blackflies and has previously been found associated with sex (Bedo, 1978).

The complex rearrangement labelled $I L$ Q.R.S.T was found 16 times, but only as the heterozygote, and only in males from Norfolk. None of the individual inversions which make up $I L-Q . R . S . T$ were found independently. The constituent inversions $I L-Q$ and $R$ are distal, nonoverlapping, and distinctive. However, the basal overlapping part of the complex forms a tangled heterozygous loop which varies between individuals, and it is not clear whether this is due to variation in the number of inversions linked in the complex, or variation in the sequence of the other homologue. However, the basal overlapping complex consists of at least two inversions $(I L-S$ and $T$ ) and probably more. It is possible that $I L-A$ might be included. IL-Q.R.S.T is shown in fig. 4 .

The inversion $I S-P$ (fig. 5) was found in six larvae taken from Carlton Forehoe, and also in one additional larva from Hingham. The inversion $I S-H$ (fig. 5) was found in a total of four larvae taken from Carlton Forehoe and Hingham. On each occurrence $I S$ - $H$ was linked to $I S$ - $P$. Neither inversion was found in the homozygous state, and all seven larvae were males which also possessed the heterozygous complex IL-Q.R.S.T.

It is concluded that $I S$-split is due to genetic differences between the $\mathrm{X}$ and $\mathrm{Y}$ chromosomes and represents the primary differential region of the sex chromosomes, including or very close to the primary sex determining locus. The single heterozygous female is enigmatic, but could result from an exceptional crossover between $I S$-split and the primary sex determining locus, or be the result of some rare mutation. Alternatively, one can never entirely rule out the possibility that specimens might have been mislabelled. IL-Q.R.S.T appears to mark the $\mathrm{Y}$ chromosome. Its distribution between males and females $(16: 0)$ is 

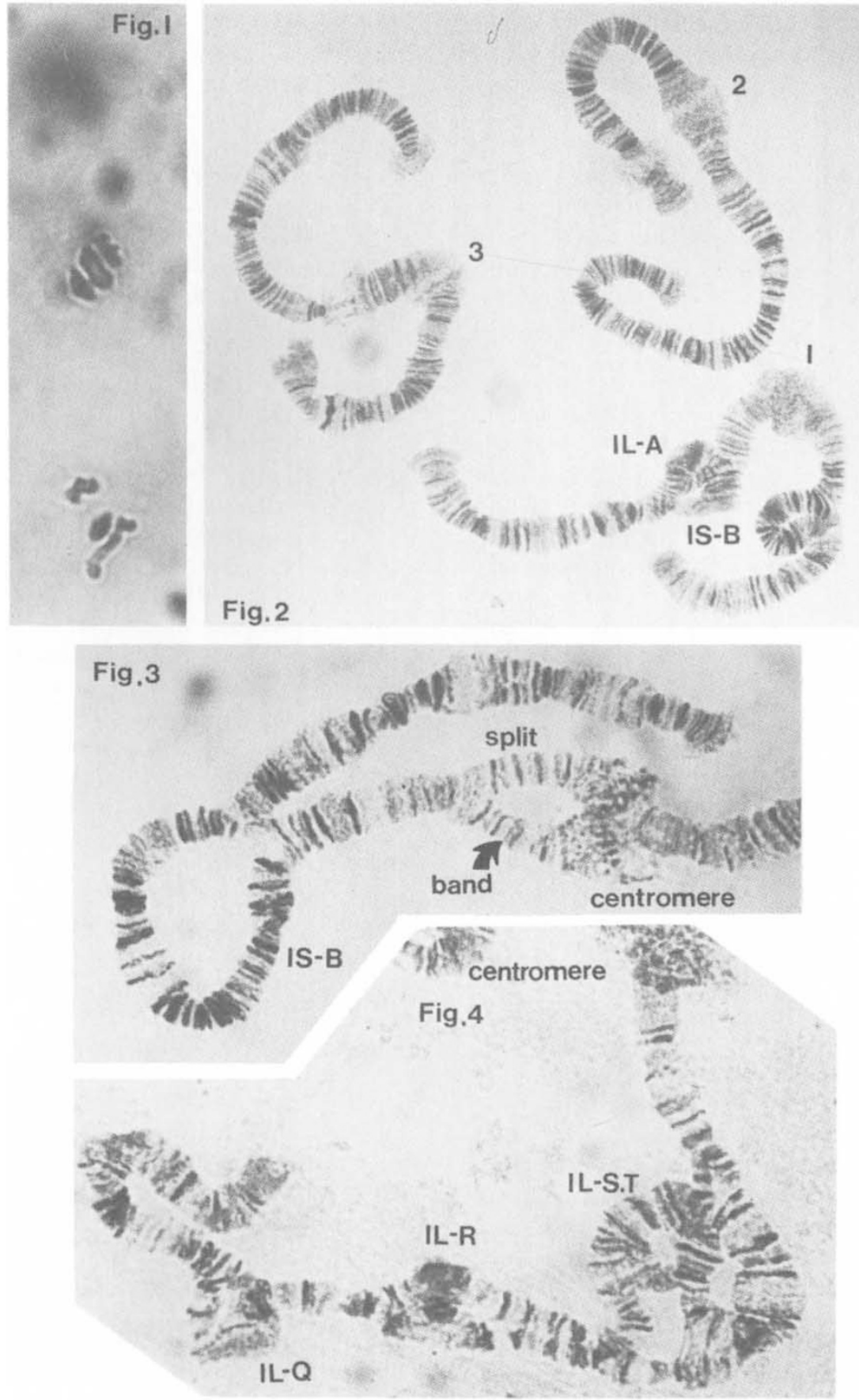

Figure 1 Testicular meiosis from last larval instar $S$. erythrocephalum.

Figure 2 Entire polytene karyotype from female larval salivary gland.

Figure $3 I S-B / S t$ from male, showing basal non-pairing region including band-staining dimorphism.

Figure $4 I L-Q \cdot R, S, T /$ St from male $S$, erithrocephalum. 

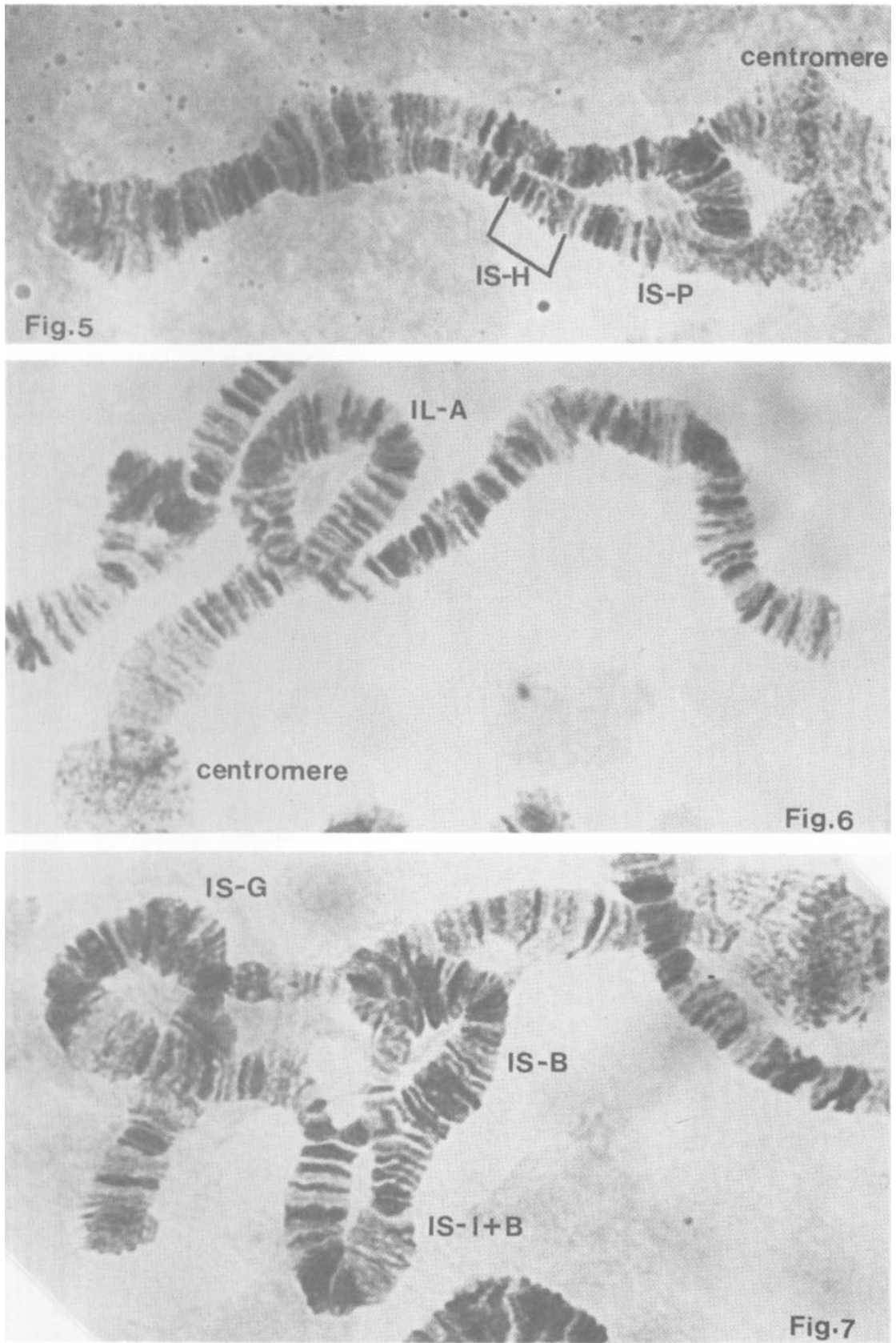

Figure $5 \quad I S-P . H / S t$ from male $S$. erythrocephalum.

Figure $6 \quad I L-A / S t$ from male.

Figure 7 IS G.I/B from male.

significantly different from the observed Norfolk sex ratio $(157: 200)$ and the observed sex distribution of $I L-A(155: 103$, see below). If $I L-Q . R . S . T$ marks the $\mathrm{Y}$-chromosome, then, presumably, $I S$ - $P$ and $I S-H$ do also.
Thus, there seems to be a pattern of serial differentiation of the sex bivalent (fig. 8). IS -split represents the primary differential region, including, or at least very close to the primary sex determining gene. The linkage of $I L-Q . R . S . T, I S-P$ and 


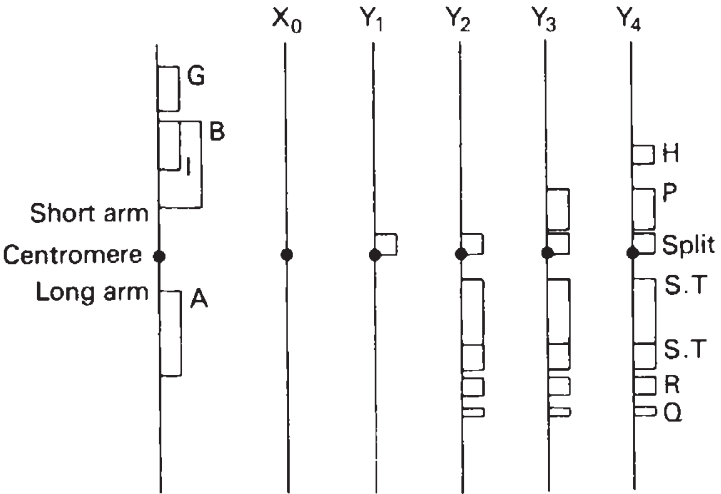

Figure 8 Idiograms of $S$. erythrocephalum first-chromosome showing sex-chromosome differentiation.

$I S-H$ to the $\mathrm{Y}$-chromosome results in the serial elimination of recombination along the sex chromosomes out from the primary sex determining locus, thus effectively increasing the size of the sex chromosome differential region.

\section{THE GENETICS OF INVERSION IL-A}

The polymorphic inversion $I L-A$ (fig. 6) was found at all seven sample sites (table 1 ) and its frequency did not vary geographically $\left(\chi_{6}^{2}=10 \cdot 19, P=0 \cdot 12\right)$. Furthermore, there is no evidence for temporal variation in inversion frequency between the 13 samples from Calton Forehoe (combining samples 10478 and $\left.5578 ; \chi_{11}^{2}=13 \cdot 24, P=0 \cdot 28\right)$. However, considering all 17 sexed samples, in $15 \mathrm{IL}-A$ is at a higher frequency in males than females (data not shown) and this is significantly different from the random expectation of $8.5\left(\chi_{1}^{2}=9.94, P<0.01\right)$.

The karyotype frequency distribution in the two sexes at Carlton Forehoe during 1978 (table 2) shows a general excess of heterozygotes compared with Hardy-Weinberg expectation, although this is rarely significant in individual samples with the numbers obtained. However, considering the total data (table 2) there is a significant excess of male heterozygotes $\left(\chi_{2}^{2}=14.81, P<0 \cdot 001\right)$, but not females $\left(\chi_{2}^{2}=1 \cdot 40, P>0 \cdot 20\right)$, and this excess occurs only in males from "early" samples $\left(\chi_{2}^{2}=\right.$

Table 2 Karyotype frequency data for Carlton Forehoe

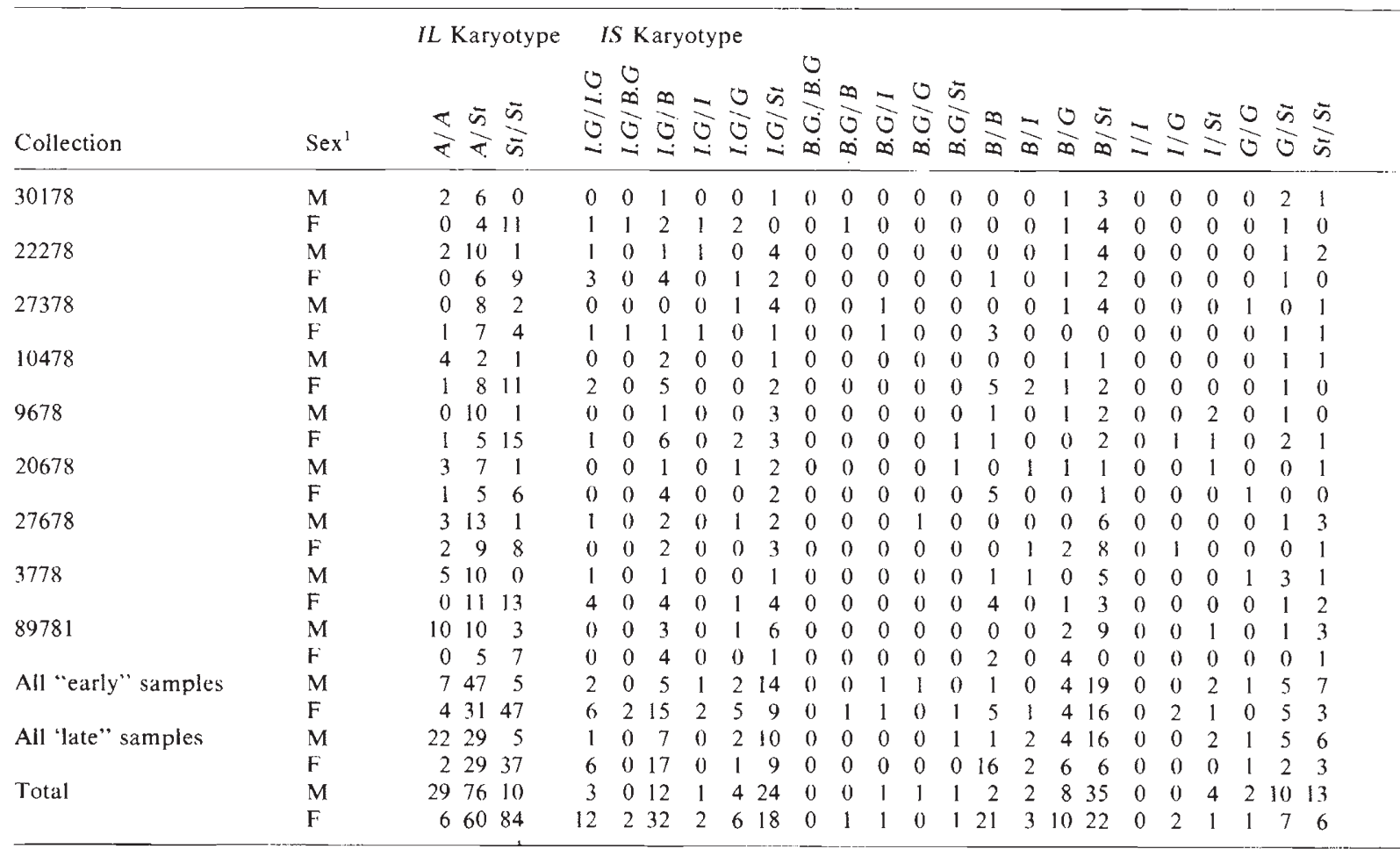

1. It was not always possible to determine larval sex. Such animals are included in table 1 but not table 2 . 
$20 \cdot 89, \quad P<0.001)$ and not males from "late" samples $\left(\chi_{2}^{2}=1 \cdot 11, P>0 \cdot 20\right)$.

The excess of heterozygotes and higher frequency of $I L-A$ in males could result from a higher frequency of $I L-A$ on the Y-chromosome, as opposed to the $\mathrm{X}$-chromosome. The frequency of $I L$ - $A$ on the $\mathrm{X}$-chromosome $\left(P_{x A}\right)$ is given by the frequency in females; and the frequency on the Y-chromosome, $P_{y A}=$ Twice the frequency in males- $P_{x A}$. The expected male karyotype frequency distribution for each generation, calculated using these sex-chromosome inversion frequencies, is a much better fit than is obtained by assuming equal frequencies on the $\mathrm{X}$ and $\mathrm{Y}$ chromosomes (table 3), except for the fifth generation; but this might be expected, because the fifth generation is only represented by a single "late" sample.

To summarise, there is evidence that heterozygosity for inversion $I L-A$ is correlated with faster larval development of males, but not necessarily females. Furthermore, $I L-A$ occurs at a higher frequency on the Y-chromosome, as opposed to the $\mathrm{X}$ chromosome, and this results in an overall excess of male heterozygotes.

\section{THE GENETICS OF IS-B.I.G}

The polymorphic inversion $I S-I$ is tandem and included in $I S-B$, which is proximal and does not overlap the distal polymorphic inversion $I S-G$ (see figs. 7 and 8). However, these inversions were found in linkage disequilibrium. The chromosome sequences $I S$-B.I and $I S-B . I . G$ were not found at all, and inspection of Table 1 indicates a deficiency of $I S-B . G$.

The sample frequencies of the $I S$ chromosome sequences (table 1) reveal no evidence for temporal variation between the 13 samples from Carlton Forehoe (combining samples 10478 and 5578 , and sequences B.G, I and $S t ; \chi_{33}^{2}=32 \cdot 70$, $P=0 \cdot 5)$, but suggest geographic variation between the seven sites (using total Carlton Forehoe data and combining sequences B.G, I and $S t ; \chi_{18}^{2}=$ 58.33, $P<0.0001)$. Furthermore, in all 17 sexed samples $I S$-St was at higher frequency in males than females (data not shown), and this is significantly different from the random expectation of $8.5\left(\chi_{1}^{2}=8.5, P<0.001\right)$. Similarly, $I S-B$ was found at higher frequency in 13 samples, lower frequency in three and equal in one; and this is a significant departure from random expectation $\left(\chi_{1}^{2}=5 \cdot 88, P<0 \cdot 02\right)$.

With six observed $I S$-sequences there are 21 possible different karyotypes for each sex. These are shown in table 2 , from which inversion frequencies for males and females at Carlton Forehoe can be derived separately. Using total data the frequencies are (males/females): IS-I.G, $0 \cdot 19 / 0 \cdot 28 ; I S-B$, $0 \cdot 25 / 0 \cdot 37 ; I S-S t, 0 \cdot 41 / 0 \cdot 21 ; I S-G, 0 \cdot 11 / 0 \cdot 9$; and $I S-I+B . G, 0 \cdot 04 / 0 \cdot 05$. The sex differences for $I S$ I.G, IS-B and IS-St can be tested by chi-square and are significant in each case at the 1 per cent level. Furthermore, in males, but not females, there is a general excess of heterozygote karyotypes compared with Hardy-Weinberg expectation (table 2: total males, homozygotes $\mathrm{V}$. heterozygotes, $\chi_{1}^{2}=7 \cdot 98, P<0 \cdot 01 ;$ total females, $\chi_{1}^{2}<0 \cdot 001, \mathrm{P}>$ $0 \cdot 99$ ). The excess of male heterozygotes, and sex

Table 3 Expected male $I L$-karyotype frequency distribution

\begin{tabular}{|c|c|c|c|c|c|}
\hline \multirow[b]{2}{*}{ Generation } & \multirow[b]{2}{*}{ Group } & \multicolumn{4}{|c|}{ Karyotype } \\
\hline & & $A / \ddot{A}$ & $A / S t$ & $S t / S t$ & $\chi_{2}^{2}$ \\
\hline \multirow[t]{4}{*}{1} & Observed males & 8 & 26 & 4 & \\
\hline & Observed females & 2 & 25 & 35 & \\
\hline & Expected males $\left(P_{A}=0.553\right)$ & $11 \cdot 6$ & $18 \cdot 8$ & $7 \cdot 6$ & $5 \cdot 58$ \\
\hline & Expected males $\left(P_{y A}=0.871, P_{x A}=0.234\right)$ & $7 \cdot 7$ & $26 \cdot 6$ & $3 \cdot 7$ & 0.05 \\
\hline \multirow[t]{4}{*}{2} & Observed males & 3 & 17 & 2 & \\
\hline & Observed females & 2 & 10 & 21 & \\
\hline & Expected males $\left(P_{A}=0.523\right)$ & $6 \cdot 0$ & $11 \cdot 0$ & $5 \cdot 0$ & $6 \cdot 57$ \\
\hline & Expected males $\left(P_{y A}=0.833, P_{x A}=0.212\right)$ & $3 \cdot 9$ & $15 \cdot 2$ & $2 \cdot 9$ & 0.70 \\
\hline \multirow[t]{4}{*}{3} & Observed males & 8 & 23 & 1 & \\
\hline & Observed females & 2 & 20 & 21 & \\
\hline & Expected males $\left(P_{A}=0.609\right)$ & $11 \cdot 9$ & $15 \cdot 2$ & $4 \cdot 9$ & $8 \cdot 38$ \\
\hline & Expected males $\left(P_{v A}=0.940, P_{x A}=0.279\right)$ & $8 \cdot 4$ & $22 \cdot 2$ & 1.4 & $0 \cdot 16$ \\
\hline \multirow[t]{4}{*}{5} & Observed males & 10 & 10 & 3 & \\
\hline & Observed females & 0 & 5 & 7 & \\
\hline & Expected males $\left(P_{A}=0.652\right)$ & $9 \cdot 8$ & $10 \cdot 4$ & $2 \cdot 8$ & 0.03 \\
\hline & Expected males $\left(P_{y A}=1.000, P_{x A}=0.208\right)$ & $4 \cdot 9$ & $18 \cdot 1$ & 0 & $11 \cdot 93$ \\
\hline
\end{tabular}




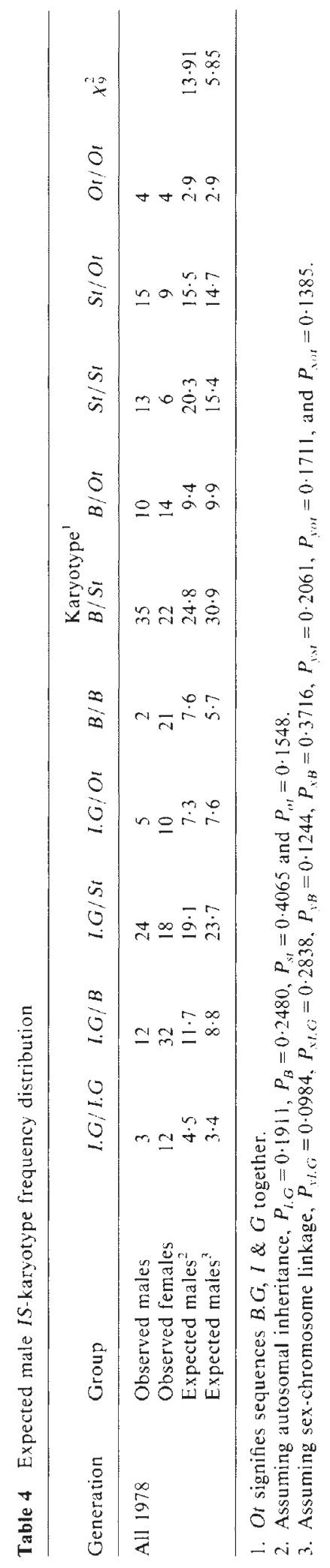


difference in inversion frequencies suggest that the inversions occur at different frequencies on the $\mathrm{X}$ and $\mathrm{Y}$ chromosomes, and karyotype frequencies expected on this assumption give a closer fit to the observed data than autosomal Hardy-Weinberg expectations (table 4).

There were differences observed between "early" and "late" samples in the frequency of the six chromosome sequences in total females, but not in males (table 2, combining IS-B.G and $I$; males, $\chi_{4}^{2}=0.52, P=0.97$; females, $\chi_{4}^{2}=13 \cdot 22$, $P=0.01)$. Sequence $I S-B$ is responsible for most of this variation, and this inversion shows an excess of $I S-B / B$ homozygotes in total females from "late" samples compared with "early" samples (analysis not shown, but see table 2 for data).

In summary, the inversions of the $I S-B+I+G$ system show linkage disequilibrium with each other and with the sex chromosomes, such that males are more frequently heterozygous than females. Furthermore, $I S-B$ seems to be correlated with speed of larval development such that $I S-B / B$ homozygote females, but not necessarily males, which are rare, develop more slowly.

\section{DISCUSSION}

Males of most species of blackfly, including $S$. erythrocephalum, are chiasmate with largely isomorphic sex chromosomes. This suggests that sex is primarily determined by a single locus, or at most a short length of chromosome. In S. erythrocephalum the primary sex chromosome differential region is the non-pairing region at the base of the short arm of the first chromsome, but this primary region can be enlarged by the serial linkage of inversions out along the chromosome. This pattern is quite common in blackflies (for references see Post, 1982a) and might be explained by the progressive linkage of new advantageous inversions to the sex locus. If such inversions include the sex locus, they will increase in frequency, but will be unable to cross over onto the other sex chromosome, even if they are advantageous in both sexes. Similarly, neutral inversions which happen to include the sex locus will be able to increase in frequency only on the sex chromosome on which they first appeared. Alternatively, the observed pattern of serial sex chromosome differentiation by inversion might be the result of selection for decreased recombination between $X$ and $Y$. Charlesworth and Charlesworth (1980) have shown that there will be selection for decreased recombination between the sex locus and a second selected locus if there is already a selectively maintained sex difference in allele frequency at the second locus. The existence of a series of such loci along the chromosome would result in the selection of inversions to progressively increase the size of the sex chromosome differential segment. This sort of selection might also explain the observed sex differences in the $I L-A$ and $I S-B+I+$ $G$ inversion systems in $S$. erythrocephalum.

To give the observed karyotypes, $I L-A$ and $I S-B+I+G$ must be able to recombine with the sex chromosomes, although the rate of recombination is unknown. These inversions are polymorphic throughout southern England and $I S-B+I$ (at least) have been found in populations around Leningrad (Petrukhina, 1968). Because they are widespread, these inversions are probably not new and hence the sex differences in frequencies should have had time to decay if they were not maintained by selection. The nature of this presumed selection is not clear but could be involved with the inversions' apparent effects on speed of larval development.

Males which emerge as adults earlier have more potential mates (Wiklund and Fagerstrom, 1977), which could give the $I S-A / S t$ males an advantage, because they are faster developing than the homozygotes. This male limited heterozygote advantage could maintain the $I L-A$ polymorphism, but not the sex difference in frequency; for this there must also be selection on the female (Charlesworth and Charlesworth, 1980). Faster developing females are smaller and have fewer ovarioles (Ruhm and Hechler, 1974) but there is no evidence to suggest that $I L-A$ is associated with speed of development in female $S$. erythrocephalum. However, $I S-B / B$ females do develop more slowly and this is likely to be selected. To maintain the observed sex difference in frequency of $I S-B$ there must also be selection in the males. It is possible that $I S-B / B$ males also develop more slowly, but such male karyotypes are rare and it was not possible to test this hypothesis with the sample sizes obtained.

\footnotetext{
Acknowledgements I am especially pleased to thank Dr G. M. Hewitt for his supervision of this work. I am also grateful to Mr R. C. Buxton for permission to work at Carlton Forehoe. J. Gauckwin and C. P. Post helped to prepare the manuscript. The work was supported by the Natural Environment Research Council.
} 


\section{REFERENCES}

BEDO, D. C. 1978. Band and nucleolar polymorphisms in polytene chromosomes of Simulium ornatipes (Diptera: Simuliidae). Cytobios, 21,113-133.

('HARLESWORTH, B. 1978. Model for evolution of Y chromosomes and dosage compensation. Proc. Natl. Acad. Sci., $75,5618-5622$.

('HARLESWORTH, D. AND CHARI.ESWORTH, B. 1980. Sex differences in fitness and selection for centric fusions between sex-chromosomes and autosomes. Genet. Res., 35 , 205-214.

I)ARLINC:TON, ( . I). ANI) I.A (OUR, L. I: 1976. The Handling of Chromosomes. Allen and Unwin, London.

MULLER, H. J. 1914. A gene for the fourth chromosome of Drosophila. J. Exp. Zool, 17, 325-336.

NEI, M. 1970. Accumulation of nonfunctional genes on sheltered chromosomes. Am. Natur., 104, 311-322.

PETRUKHINA. T. F. 1968. A study of chromosomal polymorphism in a natural population of the Red-Headed Fly Boophthora erythrocephala. Tsitologiva, 10, 1148-1154.
POSI, R. J. 1982a. Sex-linked inversions in blackflies (Diptera: Simuliidae) Heredity, 48, 85-93.

POST, R. J. $1982 \mathrm{~b}$. Notes on the natural history of Simulium erythrocephalum (Diptera: Simuliidae). Ent. Mon. Mag., $118,31-35$.

POST, R. J. 1983. The annual cycle of Simulium erythrocephalum (Diptera: Simuliidae) at a site in Norfolk. Freshwater Bio$\log y, 13,379-388$.

PURI, I, M. 1925. On the life history and structure of the early stages of Simuliidae (Diptera: Nematocera), Part 1. Parasigology, 17, 295-334.

ROTHFFL, K. H. AND MASON, G. F. 1975. Achiasmate meiosis and centromere shift in Eusimulium aureum (DipteraSimuliidae). Chromosoma, 15, 111-124.

RUHM, W. ANI) HI: (HI.:R, J. 1974. Untersuchungen über die potentielle Natalität vershiedener mammalophiler Kriebelmückenarten unter besonderer Berücksichtigung von Boophthora erythrocephala De Geer. Z. Ang. Ent., 77, 1931.

WIKLUND, C. AND FAGERSIROM, T. 1977. Why do males emerge before females? Oecologia, 31, 153-158. 